\title{
Persistence homology of entangled rings
}

\author{
Fabio Landuzzi $\odot,{ }^{1}$ Takenobu Nakamura $\odot{ }^{2,3}$ Davide Michieletto $\odot,{ }^{4,5,6}$ and Takahiro Sakaue $\oplus^{1,2, *}$ \\ ${ }^{1}$ Department of Physics and Mathematics, Aoyama Gakuin University, 5-10-1 Fuchinobe, Chuo-ku, Sagamihara, Japan \\ ${ }^{2}$ PRESTO, Japan Science and Technology Agency (JST), 4-1-8 Honcho Kawaguchi, Saitama 332-0012, Japan \\ ${ }^{3}$ National Institute of Advanced Industrial Science and Technology (AIST), 1-1-1, Umezono, Tsukuba, Ibaraki 305-8568, Japan \\ ${ }^{4}$ School of Physics and Astronomy, University of Edinburgh, Peter Guthrie Tait Road, Edinburgh, EH9 3FD, United Kingdom \\ ${ }^{5}$ MRC Human Genetics Unit, Institute of Genetics and Molecular Medicine, University of Edinburgh, \\ North Crewe Road, Edinburgh, EH4 2XU, United Kingdom \\ ${ }^{6}$ Centre for Mathematical Biology and Department of Mathematical Sciences, University of Bath, \\ North Road, Bath, BA2 7AY, United Kingdom
}

(Received 22 May 2020; accepted 28 August 2020; published 30 September 2020)

\begin{abstract}
Topological constraints (TCs) between polymers determine the behavior of complex fluids such as creams, oils, and plastics. Most of the polymer solutions used in everyday life employ linear chains; their behavior is accurately captured by the reptation and tube theories which connect microscopic TCs to macroscopic viscoelasticity. On the other hand, polymers with nontrivial topology, such as rings, hold great promise for new technology but pose a challenging problem as they do not obey standard theories; additionally, topological invariance, i.e., the fact that rings must remain unknotted and unlinked if prepared so, precludes any serious analytical treatment. Here we propose an unambiguous, parameter-free algorithm to characterize TCs in polymeric solutions and show its power in characterizing TCs of entangled rings. We analyze large-scale molecular dynamics simulations via persistent homology, a key mathematical tool to extract robust topological information from large datasets. This method allows us to identify ring-specific TCs which we call "homological threadings" (H-threadings) and to connect them to polymer behavior. It also allows us to identify, in a physically appealing and unambiguous way, scale-dependent loops which have eluded precise quantification so far. We discover that while threaded neighbors slowly grow with ring length, the ensuing TCs are extensive also in the asymptotic limit. Our proposed method is not restricted to ring polymers and can find broader applications for the study of TCs in generic polymeric materials.
\end{abstract}

DOI: 10.1103/PhysRevResearch.2.033529

\section{INTRODUCTION}

Ring polymers are the simplest class of topologically nontrivial polymers that manifestly depart from the predictions of theoretical cornerstones such as the reptation and tube models [1]. Over the last three decades there have been several theoretical and experimental attempts at understanding the statics and dynamics of rings [2-19] and yet their behavior in entangled solutions is still poorly understood. Individual ring polymers in the melt or entangled solutions assume compact non-Gaussian conformations, which are distinct from the ones assumed by linear polymers. Understanding the topological origin of these conformations and their consequence on the ring entanglements and ensuing dynamics of the bulk is crucial, not only from a fundamental perspective but also for practical applications such as the rheology of ring polymer melts [8], the phase behavior of blends [20-23], and even

\footnotetext{
*sakaue@phys.aoyama.ac.jp

Published by the American Physical Society under the terms of the Creative Commons Attribution 4.0 International license. Further distribution of this work must maintain attribution to the author(s) and the published article's title, journal citation, and DOI.
}

the dynamical organization of chromosomes in the cell nucleus [24].

Due to their unconventional conformations in equilibrium, long nonconcatenated rings in the melt experience topological constraints (TCs) that are markedly different from ordinary entanglements between linear chains. Early models for ring polymers pictured them in crumpled, double-folded lattice animal conformations $[4,7,25,26]$, which imply selfsimilar fractal conformations and dynamics, very different from the reptation of entangled linear polymers. More recent models relaxed the assumption of strict double-folded states $[12,27,28]$ and studied the appearance of transient opening of the double-folded structure thereby forming loops [27]. These local openings can themselves accommodate doublefolded contour of the same or other neighboring rings; these "threadings" do not violate the topological invariance of the system and have been found to be abundant in entangled solutions of rings $[15,29,30]$. Threading of rings is an architecture-specific TC that is not present in systems of linear chains and gives rise to unique dynamical states; for instance, interchain threadings can slow down rings in the bulk [5,8,30], yield a heterogeneous, glasslike dynamics [31-33], and cause an anomalous response to extensional flow [17,34], while intrachain threadings (self-threading) can dramatically increase the relaxation time of rings in dilute conditions $[16,35]$. 
While it is now clear that threading between rings plays a crucial role in the dynamics of rings, its unambiguous identification remains far from trivial. The reason for this is that two rings that are mutually threaded are topologically equivalent to nonthreaded ones. At present, three methods have been proposed to detect threading in solutions of rings: (i) using a local deformation of their contours and a background mesh [29], (ii) reducing their contours to primitive paths and using geometrical analysis on these paths [15], and (iii) calculation of their minimal surface and analysis of intersections [36]. Albeit these methods give qualitatively similar results, they are cumbersome to implement and require arbitrary choices of parameters. Additionally, they are ring-specific and cannot be mapped onto systems of linear chains, thus preventing a direct comparison between TCs in solutions of ring and linear polymers.

To overcome this problem, in this paper we propose an unambiguous and parameter-free algorithm that employs persistence homology (PH) [37-39]. We apply this algorithm to datasets from molecular dynamics (MD) simulations of entangled solutions of rings and show that it allows us to define a new and broader class of TCs between ring polymers which we call "homological threadings," or H-threadings. Unlike previous definitions of threadings, an H-threading is defined by the homological property of the system, and its identification is unique. Persistent homology provides us with a powerful tool to extract useful information hidden in the big data obtained from, e.g., large-scale MD simulations of polymer melts $[6,40]$ or $x$-ray tomography of elastic bands [18]. Additionally, it has several advantages over other methods: (i) the ability to detect and quantify so-far elusive hierarchical loops in the folding of rings, (ii) very efficient and stable numerical (open-source) implementation, (iii) uniqueness of the analysis result (no free parameters), and (iv) it can be readily generalized to detect higher-dimensional structures in data sets.

Importantly, while hierarchical loops are now central in state-of-the-art models to describe the static and dynamic properties of ring polymers in dense solutions [12,27], their characterization remains very poor due to a lack of algorithms that can detect them. Here we show that the $\mathrm{PH}$ analysis is perfectly positioned to overcome this current limitation. Finally, while in this work we focus on entangled ring polymers, our method may be employed more broadly to characterize TCs in generic polymeric materials.

\section{MATERIALS AND METHODS}

\section{A. Molecular dynamics simulations}

Here we analyze MD simulations reported in previous works (the interested reader may find more details in Refs. [31,32]). Briefly, the polymers are modelled as KremerGrest chains made of beads of size $\sigma$. The beads are connected along the backbone by finite-extension nonlinearelastic (FENE) bonds, and interactions between beads are governed by the Weeks-Chandler-Andersen (WCA) potential (purely repulsive Lennard-Jones). We also introduce polymer stiffness by including a Kratky-Porod term acting on triplets of neighboring beads $U_{\text {bend }}=\left(k_{B} T l_{p} / \sigma\right)(1+\cos \theta)$, where $\theta$ is the angle formed by consecutive bonds and $l_{p}=5 \sigma$.
The motion of the beads is evolved via a Langevin equation that couples the motion of the beads with an implicit solvent. The damping constant is set to $\gamma=\tau_{M D}^{-1}$, where $\tau_{M D}=$ $\sqrt{m \sigma^{2} / \epsilon}$ is the microscopic characteristic time. The thermal noise satisfies the fluctuation dissipation theorem, and to integrate the equations of motion we employ a velocity-Verlet algorithm with time step $d t=0.01 \tau_{M D}$.

\section{B. Persistence homology algorithm}

Using configurations from MD simulations as an input data, the PH analysis generates a diagram called the persistent diagram (PD), from which we can extract hidden topological features [37-39]. For our present purpose, let us consider the $m$ th ring $(m \in[1, M])$; the input data is $\left\{\vec{r}_{n}^{(m)}\right\}=$ $\left(\vec{r}_{1}^{(m)}, \vec{r}_{2}^{(m)}, \ldots, \vec{r}_{N}^{(m)}\right)$, where $\vec{r}_{n}^{(m)}$ is the position of the $n$th monomer in the $m$ th ring. Associated to each monomer's coordinate, we assign a ball of radius $r(\alpha)=\sqrt{\alpha}$, which should not be confused with the real radius $\sigma / 2$ of the monomers. Initially we set $\alpha=0$, so the input data is regarded as a collection of volumeless points. This method could be generalized to the copolymer setting a different initial radius for different types of monomers. We then gradually inflate the balls, and for each value of $\alpha$ we connect all the balls that are even partially overlapping. This algorithm generates sets of topological structures made of connected balls that evolve with the balls' radius $r(\alpha)$.

\section{DISCUSSION}

\section{A. Persistent homology}

Persistent homology is a method to compute the topological features of a system at different length scales [37-39]. The main concepts in this type of analysis are the persistent homology point, that defines the size at which a certain topology of the system appears/vanishes (birth/death scale), and the persistent diagram (PD), a collection of all the $\mathrm{PH}$ points of fixed dimension (see Materials and Methods). Our analysis is focused on entangled solutions of unknotted, unconcatenated ring polymers; in order to capture the constraints related with the ring topology, we consider the homology of dimension 1 that identifies the formation of "loops." Importantly, here and in what follows we refer to a "loop" as a one-dimensional (1D) geometric object detected in the $\mathrm{PH}$ analysis, while the term "ring" is reserved for the full polymer contour length.

An example of this analysis is shown in Fig. 1. Starting from a single snapshot from MD simulations of ring polymers-made as sterically interacting connected beadswe associate at each bead position a sphere of size $r(\alpha)=$ $\sqrt{\alpha}$. When $\alpha<\alpha_{\min }[\simeq 0.3$ in the example of Fig. 1(bi)], there is no loop present because there are no overlapping spheres. At $\alpha=\alpha_{\min }$ all the balls are connected with the consecutive along the ring, and therefore the ring itself is identified as a loop in the PD analysis [Fig. 1(bii)]. It should be mentioned that the first loop to be identified by the $\mathrm{PH}$ algorithm is not necessarily the full ring contour length and may instead take up only a fraction of the whole ring, in agreement with previous findings using "contact maps" [27,41]. For increasing values of $\alpha$, certain existing loops are annihilated while others are formed [Figs. 1(c) and 1(d)], and eventually, 


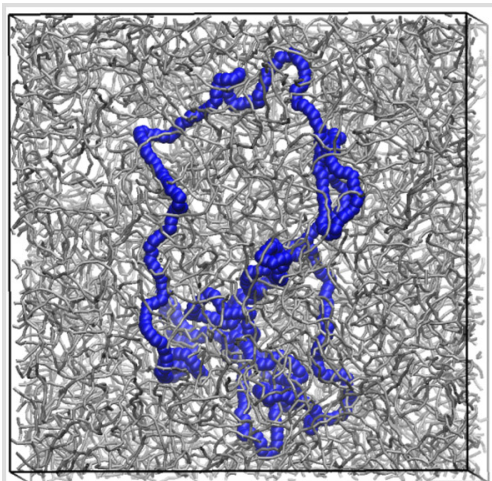

(a)

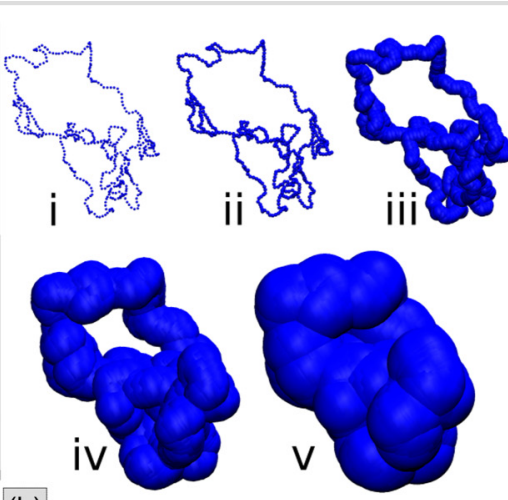

(b)

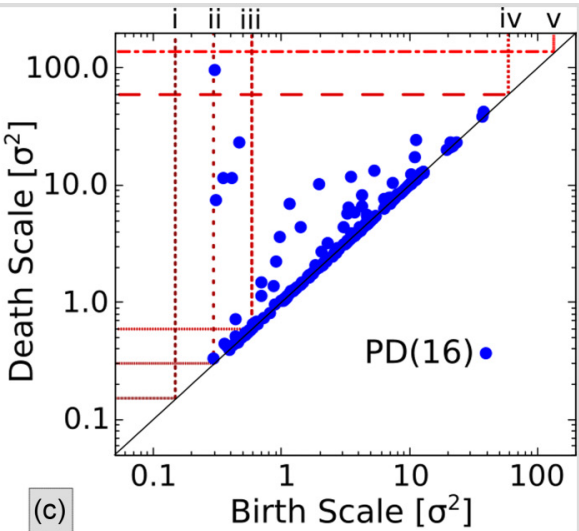

FIG. 1. Persistent diagram of an unknotted and uncatenated polymer ring composed by $N=512$ monomers. (a) Snapshot of entangled solution of unknotted and nonconcatenated rings $(N=512, M=40)$. As example, we select an arbitrary ring in this solution. (b) We consider ball representations of ring conformation with various ball radius $r(\alpha)=\sqrt{\alpha}$. (c) Persistent diagram of the ring. Birth and death scales for five initial loops $c_{k}^{(m)}$ with $k=1-5$ are $\left(b_{k}^{(m)}, d_{k}^{(m)}\right)=(0.296,0.331),(0.298,96.232),(0.305,7.393),(0.355,11.480),(0.363,0.440)$. Note the correspondence in numbering between (b) and (c)

at $\alpha \simeq 96$ for this particular example, the inner space of the ring is filled in and no loop is detected.

If a loop of connected balls exists for a given $\alpha$, a small inflation may lead to a change of topological structures, yielding the disappearance of such a loop and the appearance of others. The sequence of changes in these 1D topological features for different $\alpha$ is intimately associated to the hierarchical, multiscale folding of the rings and is encoded in the PD generated by the $\mathrm{PH}$ algorithm. Below, we explain how to translate the PD into useful information about the folding and topological interactions between rings in solution.

For a given ( $k$ th) loop on the $m$ th ring, i.e., $c_{k}^{(m)}$, its birth and death scales are denoted as $b_{k}^{(m)}$ and $d_{k}^{(m)}$ and capture the length scales at which the loop first appears and then disappears, respectively. It should be highlighted that the birth scale is also related to the maximum spatial distance between two monomers that generate a certain loop; indeed, when $\sqrt{\alpha}$ is larger than the distance of two monomers, these are then "in contact" and a loop is created by the connected portion of contour length between these two monomers. The minimum length to achieve this is $\sqrt{b_{k}^{(m)}}$. The death scale is instead related with the geometric three-dimensional (3D) size of such a loop. More precisely, $\sqrt{d_{k}^{(m)}}$ corresponds to the radius of the maximum sphere that can be drawn inside the loop, $c_{k}^{(m)}$.

The PD of the $m$ th ring is made by the collection of all the birth and death scales $\left(b_{k}^{(m)}, d_{k}^{(m)}\right)$ of all the loops generated by ranging $\alpha \in[0, \infty)$, i.e., $P D(m) \equiv$ $\left\{\left(b_{k}^{(m)}, d_{k}^{(m)}\right) \in \mathbb{R}^{2} \mid k=1,2, \ldots\right\}$. This set of points is typically represented in the top half triangle of a two-dimensional plane as in Fig. 1(c).

\section{B. Persistence diagram of crumpled rings}

For each point $\left(b_{k}^{(m)}, d_{k}^{(m)}\right)$ in the PD (see Materials and Methods), we define its life-span $l\left(c_{k}^{(m)}\right) \equiv d_{k}^{m}-b_{k}^{m}$, which measures the distance from the diagonal line to the point. The points that lie far from the diagonal, i.e., with large life-span, are the ones reflecting robust and persistent features of the system, whereas the ones lying close to the diagonal are often associated with noise [42]. Among other off-diagonal points, we call the loop associated to the point with the largest life-span "primal." More specifically, for open noncrumpled conformations, the primal loop may correspond to the ring itself, and its birth scale is expected to be on the order of $\simeq(\sigma / 2)^{2}$ ( $\sigma$ is the size of a simulated bead). In general, the primal mirrors the presence of a large opening of the doublefolded structure in the ring's conformation and its life-span quantifies the size of the opening.

For example, in Fig. 2 we show two typical conformations and their respective PDs in red (ring 17) and blue (ring 18). The primal loop of the red ring has a large death scale and its life-span is much longer than that of the blue ring (notice the logarithmic scale in the plot). This difference reflects the fact that while ring 17 displays a large opening, ring 18 assumes a more crumpled and double-folded conformation entailing a much shorter primal life-span [see the snapshot in Fig. 2(a)]. Another notable feature of Fig. 2(a) is that ring 18 displays more points away from the diagonal than ring 17 (compare, e.g., the interval $[0.5,5] \in$ birth scale). This feature of the PD reflects the crumpled nature of the conformation: the more crumpled a ring is, the more balls of a certain radius form bridges between two distal segments of the ring contour, in turn generating moderately robust loops.

Focusing our attention on the primal loop, it is possible to study the average linear size $\sqrt{d_{P L}}$ of the loop at different ring lengths, where $d_{P L}$ is the death scale of the primal loop. In Fig. 2(b), we show that this quantity follows a power-law dependence similar to the radius of gyration. This result agrees with existing theories $[9,12-14,24,43]$ that predict an exponent $1 / 2 \leqslant v \leqslant 1 / 3$, with $v=1 / 2$ for the short ring length, an intermediate regime with $v=0.4$ [43], and a compact long ring regime $v=1 / 3$ [44]. In the inset is reported the probability distribution of $\sqrt{d_{P L}}$ for different ring sizes to show that not only the average but also the peak value of the 

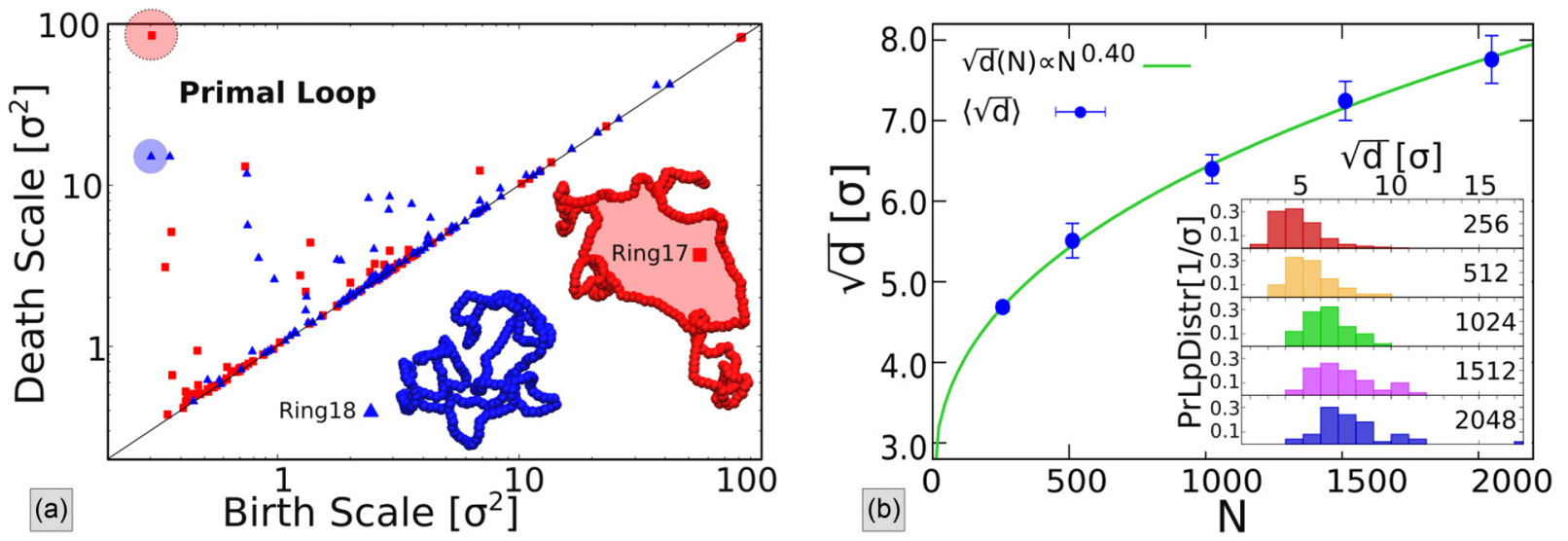

FIG. 2. Primal loop. (a) Comparison of PDs obtained from different ring conformations with $N=256$, in this case, ring no. 17 (red) and ring no. 18 (blue). The largest loop (highlighted) in the red ring has a PD point with life-span much larger than all the other loops. The crumpled conformation of the blue ring has a primal loop whose life-span is much shorter than the red one. (b) Dependence of the primal loop size (measured as the square root of its death scale $\sqrt{d_{P L}}$ ) on ring length. The data for the ring lengths $N=256,512,1024,1512,2048$ (blue dots) are fitted with a power-law curve (green curve) with exponent $v=0.40 \pm 0.11$. The inset shows the probability distribution for each ring length; notice that the peak values also show an analogous power-law dependence with $N$.

distribution is shifted towards larger sizes. For comparison, we show the probability distribution of the radius of gyration $R_{g}$ and its correlation with $\sqrt{d_{P L}}$ in Appendix A.

Having identified features of the $\mathrm{PH}$ analysis that characterize the conformation of single rings, we now aim to employ this tool to detect and quantify inter-ring interactions called threadings [29], for which an unambiguous and unique (i.e., without arbitrary and free parameters) identification algorithm is needed.

\section{Topological interactions: Homological threading}

Let us consider two unconcatenated and unlinked rings in 3D space; it is natural to define the arrangement of the two rings depicted in Fig. 3(a) as one in which the black ring is "threading" the other by crossing twice the surface spanned by the closed blue curve. Bending the blue ring into a baseball seam-like shape [Fig. 3(b)], the black ring still seems to pierce twice through the surface. In contrast, one may argue that there is no threading in Fig. 3(c), where the arrangements of the curves is the same but the spanned surface is forming a gulf that is not pierced by the former curve. Given the symmetry of the baseball seam curve, which side is selected as a spanning surface is a subtle matter. Such a subtlety would be even more enhanced for 3D crumpled rings. Additionally, while the situations in Figs. 3(b) and 3(d) appear different, they can be continuously mapped to the same three-dimensional arrangement [as Fig. 3(d)]; furthermore, the physical hindering of the black curve on the dynamics of the blue may be similar. Finally, in both cases the obstacle can be removed by a continuous deformation, for instance, by sliding it to left direction, as threadings are not conserved under isotopy. Note that Figs. 3(a)-3(d) are a typical example of isotopic change, and there can be other examples with similar ambiguity when we define threading in an intuitive way.
Nevertheless, here we aim to propose a definition for which all arrangements [Figs. 3(a)-3(e)] are identified as threadings, and because here they are discovered through a persistence homology analysis, we will dub them "homological threadings" (H-threadings).

In order to characterize the topological interaction between two rings, say $i$ and $j$, we construct four PDs from the configuration data of the two rings. Two of them are the PDs of each ring, which we write $\operatorname{PD}(i)$ and $\operatorname{PD}(j)$. The third one is the PD obtained from the union of the configurations of these two rings $\mathrm{PD}(i \cup j)$, and the last one is the union of $\mathrm{PD}(i)$ and $\operatorname{PD}(j)$, which we denote as $\operatorname{uPD}(i, j) \equiv \operatorname{PD}(i) \cup \operatorname{PD}(j)$. From these, we create the following PDs as a set difference:

$$
\begin{aligned}
& \mathrm{PD}(j \rightarrow i) \equiv \mathrm{PD}(i) \backslash \mathrm{PD}(i \cup j), \\
& \operatorname{nPD}(i, j) \equiv \mathrm{PD}(i \cup j) \backslash \mathrm{uPD}(i, j),
\end{aligned}
$$

where $\mathrm{PD}(j \rightarrow i)$ represents $\mathrm{PD}$ points which are associated to a loop in ring $i$ that is however lost in the PD in which both configurations are included. Such a loop, identified as a lost point of ring $i$, should be interpreted as a loop being threaded by ring $j$. Indeed, if any segment of ring $j$ is passing through an area that was identified as an opening in ring $i$ 's conformation, then it must represent an obstacle for the ring $i$ in the sense described in Fig. 3. By symmetry, the same applies to $\operatorname{PD}(i \rightarrow j)$, with the interchange between $i$ and $j$. These relations constitute our definition of $H$-threading events. At the same time, $\operatorname{nPD}(i, j)$ represents new points created by considering both conformations and that are absent in the union of the PDs obtained using isolated conformations; these points represent new loops that are formed when balls of a certain radius bridge segments belonging to different rings and therefore may also be considered as obstacles, restricting the conformational freedom of the individual rings $i$ and $j$.

Our definition of $\mathrm{H}$-threading respects the directionality of this interaction: points in $\operatorname{PD}(i \rightarrow j)$ identify arrangements in which ring $i$ is actively $\mathrm{H}$-threading $j$, while points in 

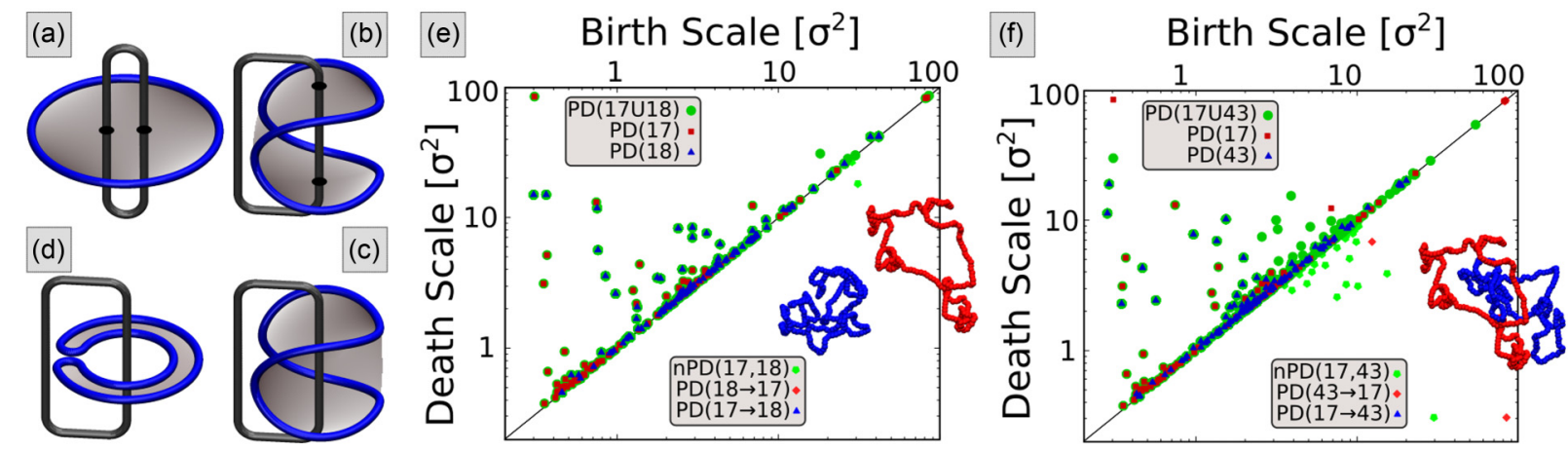

FIG. 3. Homological threading. Schematic illustrations of homological threading (left): (a) Elongated black ring is passing twice through a planar disk. (b) One side of the black rectangular ring is passing through the curved surface spanned over the baseball seam edge. (c) Configurations of bar and the baseball seam edge are the same as those in (b), but now the curved surface is spanned in a different way. (d) A side of the black ring is passing through a gulf formed by a double-folded ring. PD-based H-threading analysis (right): PDs of two rings $\mathrm{PD}(i)$ (red), $\mathrm{PD}(j)$ (blue), and $\mathrm{PD}$ obtained from the union of two rings, i.e., twin configuration $\operatorname{PD}(i \cup j)$ (green). Plot $\mathrm{E}$ shows the $\mathrm{PDs}$ of two rings ( $i=17$ and $j=18$ ) that have no H-threading between them; this is evident in the PD since all the red and blue points are overlapped by green points, i.e., there are no lost points. In the lower part of the graph are shown the new and the lost PH points, but for what was said before, no lost point and only a few new points at large scale are present (the two axis are exchanged). Snapshot at bottom right provides a visible inspection of the spatial position of the two rings. Plot $\mathrm{F}$ instead shows the ring $j=43$ that is $\mathrm{H}$-threading into $i=17$ as mirrored by the presence of lost points, i.e., the red point in the top left (primal loop of ring $i$ ) is not overlapped by a green one (the snapshot confirm this $\mathrm{H}$-threading event). The red point in the lower side corresponds to this $\mathrm{H}$-threading, and with them a cloud of new points is formed.

$\mathrm{PD}(j \rightarrow i)$ find ones in which $i$ is passively $\mathrm{H}$-threaded by $j$. To quantify these events, we can count the number $n^{(A)}$ of rings through which a reference ring is threading (active threading) and the number $n^{(P)}$ of rings threading into a reference ring (passive threading); their statistics are shown in Figs. 4(a) and 4(b). Due to symmetry, $\langle n\rangle=\left\langle n^{(A)}\right\rangle=$ $\left\langle n^{(P)}\right\rangle$, and we find that it grows with ring length as $\langle n\rangle \propto$ $N^{1 / 6}$ [or $\langle n\rangle=\ln N$, see Fig. 4(c) and discussion below]. To motivate such a scaling law, let us count the number of surrounding rings which have contacts with the reference ring. This quantity, i.e., the coordination number, is estimated as $\sim R^{3} M / V=\rho R^{3} / N \sim N^{\alpha}$, with $\alpha=3 v-1$, where $R \sim N^{v}$ is the ring spatial size. With the use of effective exponents $v=2 / 5$, valid in the intermediate crossover $N$ regime, we find $\alpha=1 / 6$. On the other hand, the compact scaling exponent $v=1 / 3$ is expected for asymptotically long rings; then we get $\alpha=0$, implying a logarithmic dependence on $N$. Smrek and Grosberg observed a similar chain length dependence for the number of rings penetrated by a single ring in their minimal surface analysis [36]. The distinction between $n^{(A)}$ and $n^{(P)}$ shows up in their distributions, with larger width for $n^{(P)}$. This may reflect the fact that passive threadings are more sensitive to the opening of loops, i.e., larger $n^{(P)}$ for rings having a primal loop with longer life-span and vice versa.

We finally stress that since $\langle n\rangle$ is the average number of $\mathrm{H}$-threadings per ring, our findings strongly suggest that our systems of rings are percolating, in the sense that all rings are connected to each other through $\mathrm{H}$-threadings.

\section{Betti number}

So far, we have analyzed the $\mathrm{H}$-threading statistics per ring as a function of ring length. However, it is natural to explore the statistics of H-threadings as a function of the loop size into which the H-threading takes place. To this end, we define the Betti number

$$
\beta^{(m)}(\alpha ; N) \equiv \int_{\alpha}^{\infty} \mathrm{d} d \int_{0}^{\alpha} \mathrm{d} b \sum_{k} \delta\left(b-b_{k}^{(m)}\right) \delta\left(d-d_{k}^{(m)}\right)
$$

for a given configuration of the $m$ th $\operatorname{ring}\left\{\vec{r}_{n}^{(m)}\right\}$ with monomer index $n \in[1, N]$.

The Betti number $\beta^{(m)}(\alpha ; N)$ counts the number of loops in the $m$ th ring if observed at the spatial resolution $\sqrt{\alpha}$. In Fig. 4(d), we compare the average Betti number per ring,

$$
\beta(\alpha ; N)=M^{-1} \sum_{m=1}^{M} \beta^{(m)}(\alpha ; N),
$$

with the quantity

$\tilde{\beta}(\alpha ; N) \equiv M^{-1} \int_{\alpha}^{\infty} \mathrm{d} d \int_{0}^{\alpha} \mathrm{d} b \sum_{k} \delta\left(b-\tilde{b}_{k}\right) \delta\left(d-\tilde{d}_{k}\right)$,

where $\left(\tilde{b}_{k}, \tilde{d}_{k}\right)$ are the birth and death scales of the loops associated with all $\mathrm{H}$-threading events, i.e.,

$$
\left(\tilde{b}_{k}, \tilde{d}_{k}\right) \in \bigcup_{i \neq j}\left\{\left(b_{l}, d_{l}\right) \in P D(j \rightarrow i)\right\} .
$$

Therefore, while Eq. (1) represents the total number of loops per ring (averaged over rings) if observed at the spatial resolution $\sqrt{\alpha}$, Eq. (2) counts only loops which are associated with an $\mathrm{H}$-threading event.

Mathematically, the set of elements $\left(\tilde{b}_{k}, \tilde{d}_{k}\right)$ is no longer strictly a PD because the right-hand side of Eq. (3) is now an union rather than a disjoint union as in the traditional definition of PD. For our analysis this distinction is practically unimportant, as we numerically checked that the multiplicity of $\mathrm{PD}$ points are unity for any ring. 

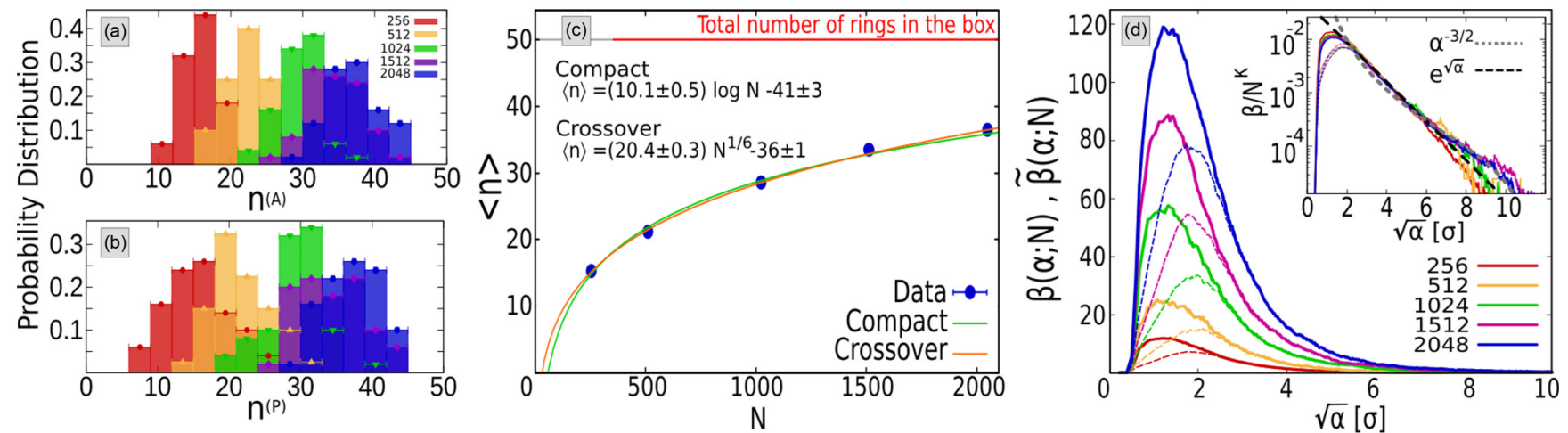

FIG. 4. Threading statistics. Probability distributions of active (a) $n^{(A)}$ and passive (b) $n^{(P)}$ threadings for various ring lengths $N$. In (c), the average of these distributions. $\langle n\rangle=\left\langle n^{(A)}\right\rangle=\left\langle n^{(P)}\right\rangle$, are shown as a function of ring length. The data are fitted with $\langle n\rangle \propto N^{1 / 6}$ (crossover $v \simeq 0.4$ ) and $\langle n\rangle \propto \log N$ (compact $v=1 / 3$ ). Notice that these values are computed as number of $\mathrm{H}$-threadings per single ring, meaning that every one of our longest rings are H-threading more than half other rings in the box. (d) The Betti number is another measure to obtain information on TCs. In the main plot we show the Betti number for isolated rings (solid curves) $\beta(\alpha ; N)$ as calculated from the PD of ring conformations for various ring lengths $N$ and we compare it with the quantity $\tilde{\beta}(\alpha ; N)$ calculated from Eq. (2) (dashed lines, see text for details). The inset shows a log-linear plot of the same curves rescaled by the factor $\left(N / N_{0}\right)^{\kappa}$. After the peak, all the data are well described by the fit $\beta(\alpha ; N)=\left(\frac{N}{N_{0}}\right)^{\kappa} \exp \left(-\sqrt{\frac{\alpha}{\alpha_{0}}}\right)$ (black dashed line) with $\kappa=1.2 \pm 0.1, N_{0}=12 \pm 3$, and $\alpha_{0}=1.4 \pm 0.2$. The collapse of these curves suggests that (H-threaded) loops display a superlinear dependence on $N$ and an exponential decay with $\alpha$. Also shown is a power-law function $f(\alpha) \sim \sqrt{\alpha}^{-3}$ (gray dotted curve); see a discussion in Appendix B for the loop size statistics.

By comparing these two quantities [see Fig. 4(d), solid and dashed lines, respectively] one can notice the following: (i) The number of threaded loops approaches the total number of loops from below, and these two quantities converge at large enough $\alpha$. (ii) The scaling function $f(\alpha) \sim e^{-\sqrt{\alpha / \alpha_{0}}}$ well captures the scale dependence of the Betti number except within an uninteresting small $\alpha \sim$ monomer scale. There is a small deviation from the exponential behavior at $\sqrt{\alpha} \gtrsim 7$. This may be a signal to a self-similar loop statistics as conjectured in the loopy-globule model [12] (see also Appendix). (iii) The Betti number follows a superlinear scaling with ring length $\beta(\alpha ; N)=N^{\kappa} f(\alpha)$ with exponent $\kappa \simeq 1.2 \pm 0.1$, indicating that the number of (threaded) loops grows at least extensively with the rings' contour length [see Fig. 4(d), inset].

We note that this finding is compelling evidence for the argument that even if the number of neighboring rings $\mathrm{H}$-threaded at any one time is bounded to plateau in the asymptotic limit [Fig. 4(c)] the number of H-threaded loops (and hence of the induced TCs) increases at least linearly with the rings' contour length.

\section{CONCLUSIONS AND PERSPECTIVES}

The notion of "loop" is often invoked as a structural motif in solutions of ring polymers $[10,12,28]$, but its precise definition is far from evident. We have proposed an operational definition of "loop" and associated topological constraints, here dubbed $\mathrm{H}$-threadings, based on a persistent homology (PH) algorithm, which allows us to unambiguously quantify the statistics of loops in an entangled solution of nonconcatenated rings. We have discovered that our $\mathrm{PH}$ analysis naturally yields (i) a sublinear scaling $\sqrt{d_{P L}} \sim N^{0.4}$ of the primal loop's death scale, reflecting the known scaling statistics of rings' size $R_{g} \sim N^{2 / 5}$ in the crossover regime [43] (Fig. 2); (ii) a slow increase of H-threaded neighbors [Fig. 4(c)]; and (iii) a superlinear scaling of the Betti number- $\beta(\alpha ; N)=N^{\kappa} f(\alpha)$ with $\kappa \simeq 1.12$-which quantifies the number of loops at length scale $\sqrt{\alpha}$ [Fig. 4(d), see also Appendix B].

It is important to stress that quantifying the statistics of loops in folded rings is not trivial $[7,27,28]$; recent theories rely on the notion that loops are formed on multiple scales in crumpled rings [12] but lack a precise way to quantify their abundance and length-scale dependence. Here we provide both via a physically appealing and unambiguous method [Fig. 4(d)].

The key point of the present work is that the number of rings that are $\mathrm{H}$-threaded by any one ring increases slowly (as $N^{1 / 6}$ in the crossover regime and as $\log N$ in the asymptotic limit) with contour length [Fig. 4(c)]. This is likely because the number of neighbors is itself conjectured to saturate around the so-called Kavassalis-Noolandi number, i.e., 10-20 [7,12-14]. Indeed, we note that $\langle n\rangle$ follows a similar molecular weight dependence to that of the effective "coordination number" that has been studied in numerical simulations [7] and plays an important role in phenomenological mean-field theories $[13,14,19,23]$. On the other hand, by analyzing the behavior of the Betti number we discovered that the number of (H-threaded) loops increases at least linearly with the rings' length, in turn implying an extensive growth of threading TCs also in the asymptotic limit [Fig. 4(d), inset]. We argue that this observation is related to the fact that crumpled ring polymers, unlike the ordinary compact globules, display a very "rough" surface whereby an extensive number of the segments are exposed and prone to be in contact with the neighboring rings [9].

In closing, we list some perspectives for the proposed method. The most natural is to investigate the relation between the present results and the rheological properties of 
(a)

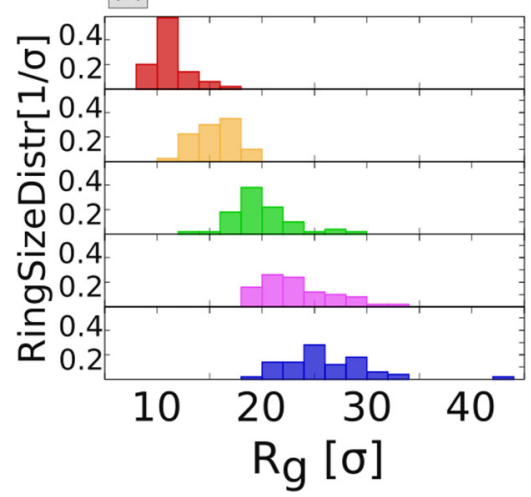

(b)

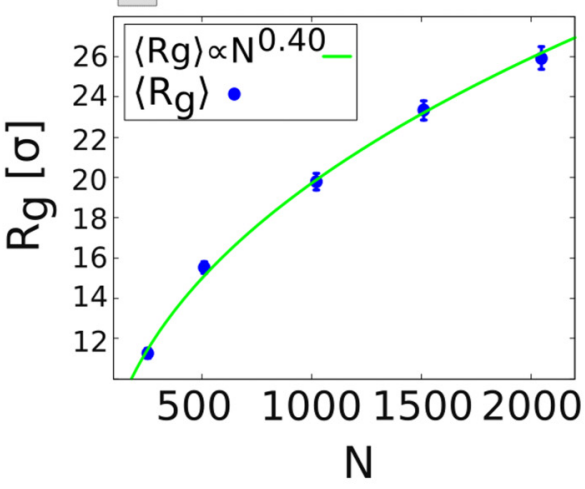

(c)

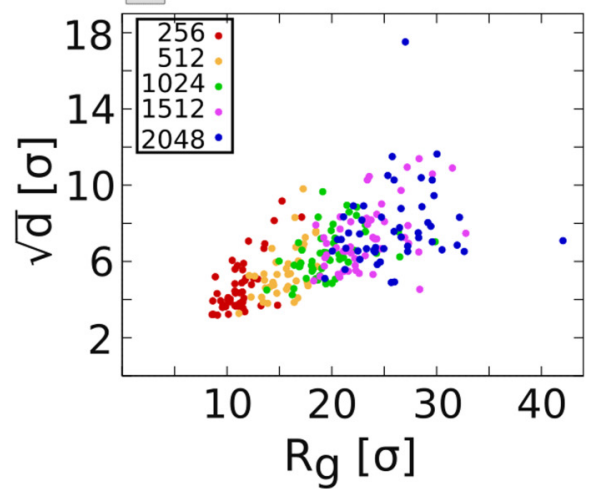

FIG. 5. Ring size. (a) Distribution of the radius of gyration $\left(R_{g}\right)$ for various ring lengths $N$ (red: $N=256$, yellow: $N=512$, green: $N=1024$, purple: $N=1512$, and blue: $N=2048$ ). (b) The average ring size scale with the same power law $\left\langle R_{g}\right\rangle \sim N^{0.4}$ as in the case of the primal loop size (see Fig. 2). (c) Primal loop size of each ring is plotted against the radius of gyration (same color code as a). The plot shows that while these two quantities are correlated on average, they represent distinct aspects of the ring shape.

the system. One way to proceed in this direction is to trace the time evolution of PDs. For instance, the time evolution of lost and new points in PDs would allow us to analyze the temporal persistence of TCs between pairs of rings and relate it to the size of the involved $\mathrm{H}$-threadings and loops. Second, our definitions of loop and H-threading can be applied to linear polymer systems too. It would be interesting to analyze entangled linear polymer solutions and see how the results compare to the present ones for ring polymers. In fact, the portability of our method to systems of polymers with other topologies is an advantage of our approach over others that are only tailored for ring polymers. Third, this method can be readily generalized to solutions of copolymers by setting a different initial radius for different types of monomers.

Finally, we would like to emphasize that the proposed method of analysis relies on the construction of PDs, such as $\mathrm{PD}(j \rightarrow i)$ and $\mathrm{nPD}(i, j)$ from a pair of original PDs. This is not a standard procedure in $\mathrm{PH}$ analysis of datasets and it is

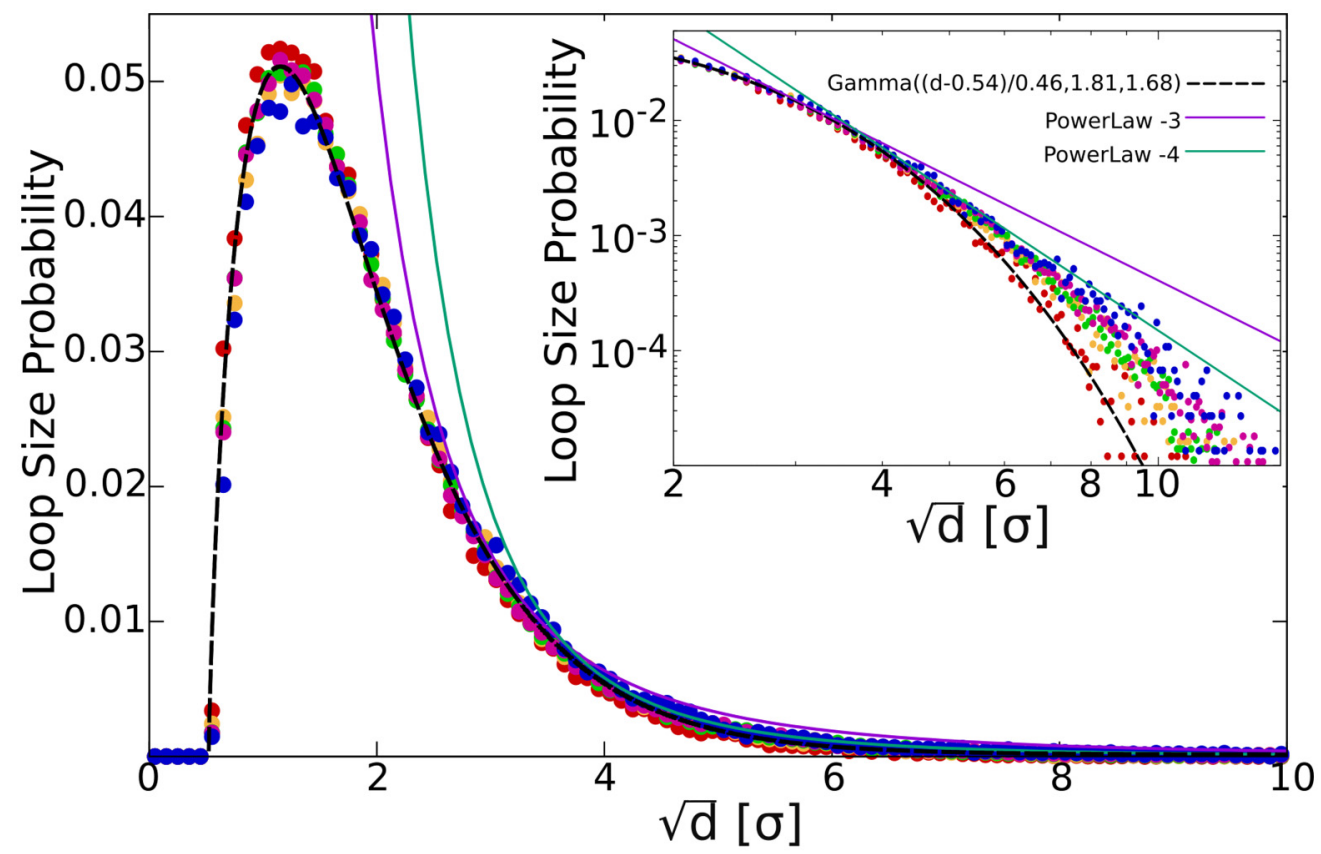

FIG. 6. Loop size distribution $\tilde{n}_{d}(r)$ at different ring lengths (red: $N=256$, yellow: $N=512$, green: $N=1024$, purple: $N=1512$, and blue: $N=2048$ ). Using the square root of the death scale as a measure of the size of a single loop, we observe that the probability to form a loop of size $\sqrt{d}$ follows the well-known gamma distribution with a shift $p(\sqrt{d})=\Gamma\left[\left(\sqrt{d}^{-3}-0.54\right) / 0.46,1.81,1.68\right]$, where 0.5 is half the average distance between single monomers. The fit captures the peak shape and the subsequent exponential decay for all the ring sizes. In the tails, the fit seems to correctly describe the short ring behavior while the longer rings experience a deviation that tend to the power-law behavior $\sim r^{-4}$. The cyan and purple lines represent a power-law fit with exponents $\sqrt{d}^{-4}$ and $\sqrt{d}^{-3}$, respectively. 
one of the key original contributions of this work. We expect that this idea has a significant potential in the broader application of persistent homology analysis, not only in polymer systems but also in other problems, e.g., to study the glass transition [39].

\section{ACKNOWLEDGMENTS}

T.S. is supported by JSPS KAKENHI (Contract No. JP18H05529) from MEXT, Japan, and JST, PRESTO (Contract No. JPMJPR16N5). T.N. is supported by JSPS KAKENHI Grant No. 15K13530 and JST-PRESTO Grant No. JPMJPR15ND, Japan. D.M. is supported by the Leverhulme Trust through an Early Career Fellowship (ECF-2019-088). The HOMCLOUD software used for the PH analysis is freely available and distributed under GPL3.

\section{APPENDIX A: COMPARISON OF PRIMAL LOOP SIZE AND RADIUS OF GYRATION}

In Fig. 5 we show the probability distribution of the gyration radius $R_{g}$ for various ring lengths. The overall trends, including the shape of distribution and its chain length dependence, are similar to those for the primal loop size [Fig. 2(b)]. This does not mean, however, the a large ring always has a large primal loop, as shown in the correlation plot in Fig. 5(c).

\section{APPENDIX B: LOOP SIZE DISTRIBUTION}

In the main text we have analyzed the loop size distribution by means of the Betti number [Fig. 4(d)],

$$
n_{\beta}(r) \equiv \beta\left(\alpha=r^{2}\right),
$$

and suggested that the large-scale part may be described by a power-law decay $n_{\beta}(r) \sim r^{-3}$. Another way to quantify the loop size distribution is to count the number of loops based on their death scale $r=\sqrt{d}$. This is because when loops with 3D size $r$ are filled up by the balls of size $\sqrt{d}$ they disappear (or die). This quantity can be readily computed from the persistence diagrams as

$$
\tilde{n}_{d}(r) \equiv \sum_{m=1}^{M} \sum_{k} \delta\left(r-\sqrt{d_{k}^{(m)}}\right) .
$$

As shown in Fig. 6, while the short-scale part of $\tilde{n}_{d}(r)$ is well-fitted by a $\Gamma$ distribution-which indicates the random distribution of the loop size-at longer scales the apparent deviation from the exponential decay shows up, and the tail seems to be better fitted by a power law with exponent $\sim-4$. This leads to a cumulative distribution

$$
n_{d}(r) \equiv \int_{r}^{R g} \tilde{n}_{d}(r) d r \sim r^{-3} .
$$

Therefore although more statistics and larger systems are necessary, our tentative conclusion is that both quantities $n_{\beta}(r)$ and $n_{d}(r)$ point to a self-similar loop size distribution conjectured in a loopy-globule model [12], i.e., decaying with the 3D size of the loop as $r^{-3}$. This should not be confused with the better-known decay of the number of loops as a function of the 1D separation as $\sim l^{-\gamma}$, with $\gamma \simeq 1.1$ for ring polymers [9]. We again emphasize that although the term "loop" is often invoked in systems of ring polymers as a crucial statistical structural element, its precise definition is far from trivial (and is hence usually not made). Through PD analysis, we have here proposed two definitions for the loop size distribution. To clarify which one is better suited for a particular purpose requires further investigation.
[1] M. Doi and S. Edwards, The Theory of Polymer Dynamics (Oxford University Press, Oxford, UK, 1988).

[2] D. J. Klein, Dynamics of entangled linear, branched, and cyclic polymers, Macromolecules 19, 105 (1986).

[3] G. B. McKenna, G. Hadziioannou, P. Lutz, G. Hild, C. Strazielle, C. Straupe, P. Rempp, and A. J. Kovacs, Dilute solution characterization of cyclic polystyrene molecules and their zero-shear viscosity in the melt, Macromolecules 20, 498 (1987).

[4] M. Rubinstein, Dynamics of Ring Polymers in the Presence of Fixed Obstacles, Phys. Rev. Lett. 57, 3023 (1986).

[5] M. Kapnistos, M. Lang, D. Vlassopoulos, W. PyckhoutHintzen, D. Richter, D. Cho, T. Chang, and M. Rubinstein, Unexpected power-law stress relaxation of entangled ring polymers, Nat. Mater. 7, 997 (2008).

[6] J. D. Halverson, W. B. Lee, G. S. Grest, A. Y. Grosberg, and K. Kremer, Molecular dynamics simulation study of nonconcatenated ring polymers in a melt, II. Dynamics, J. Chem. Phys. 134, 204905 (2011).

[7] A. Rosa and R. Everaers, Ring Polymers in the Melt State: The Physics of Crumpling, Phys. Rev. Lett. 112, 118302 (2014).
[8] Y. Doi, K. Matsubara, Y. Ohta, T. Nakano, D. Kawaguchi, Y. Takahashi, A. Takano, and Y. Matsushita, Melt rheology of ring polystyrenes with ultrahigh purity, Macromolecules 48, 3140 (2015).

[9] A. Grosberg, Annealed lattice animal model and Flory theory for the melt of non-concatenated rings: Towards the physics of crumpling, Soft Matter 10, 560 (2014).

[10] J. Smrek and A. Y. Grosberg, Understanding the dynamics of rings in the melt in terms of the annealed tree model, J. Phys.: Condens. Matter 27, 064117 (2015).

[11] A. Brás, S. Gooßen, M. Krutyeva, A. Radulescu, B. Farago, J. Allgaier, W. Pyckhout-Hintzen, A. Wischnewski, and D. Richter, Compact structure and non-Gaussian dynamics of ring polymer melts, Soft Matter 10, 3649 (2014).

[12] T. Ge, S. Panyukov, and M. Rubinstein, Self-similar conformations and dynamics in entangled melts and solutions of nonconcatenated ring polymers, Macromolecules 49, 708 (2016).

[13] T. Sakaue, Topological free volume and quasi-glassy dynamics in the melt of ring polymers, Soft Matter 14, 7507 (2018).

[14] T. Sakaue, Ring Polymers in Melts and Solutions: Scaling and Crossover, Phys. Rev. Lett. 106, 167802 (2011). 
[15] D. G. Tsalikis, V. G. Mavrantzas, and D. Vlassopoulos, Analysis of slow modes in ring polymers: Threading of rings controls long-time relaxation, ACS Macro Lett. 5, 755 (2016).

[16] B. W. Soh, A. R. Klotz, R. M. Robertson-Anderson, and P. S. Doyle, Long-Lived Self-Entanglements in Ring Polymers, Phys. Rev. Lett. 123, 048002 (2019).

[17] T. C. O'Connor, T. Ge, M. Rubinstein, and G. S. Grest, Topological Linking Drives Anomalous Thickening of Ring Polymers in Weak Extensional Flows, Phys. Rev. Lett. 124, 027801 (2020).

[18] L. R. Gómez, N. A. García, and T. Pöschel, Packing structure of semiflexible rings, Proc. Natl. Acad. Sci. 117, 3382 (2020).

[19] T. Sakaue, Statistical physics of ring polymers based on topological volume concept, React. Funct. Polym. 134, 150 (2019).

[20] R. Fitzpatrick, D. Michieletto, K. R. Peddireddy, C. Hauer, C. Kyrillos, B. J. Gurmessa, and R. M. Robertson-Anderson, Synergistic Interactions Between DNA and Actin Trigger Emergent Viscoelastic Behavior, Phys. Rev. Lett. 121, 257801 (2018).

[21] C. Chapman, S. Shanbhag, D. E. Smith, and R. M. Robertson, Complex effects of molecular topology on diffusion in entangled biopolymer blends, Soft Matter 8, 9177 (2012).

[22] Y. Zhou, K. W. Hsiao, K. E. Regan, D. Kong, G. B. McKenna, R. M. Robertson-Anderson, and C. M. Schroeder, Effect of molecular architecture on ring polymer dynamics in semidilute linear polymer solutions, Nat. Commun. 10, 1 (2019).

[23] T. Sakaue and C. H. Nakajima, Miscibility phase diagram of ring-polymer blends: A topological effect, Phys. Rev. E 93, 042502 (2016).

[24] J. D. Halverson, J. Smrek, K. Kremer, and A. Grosberg, From a melt of rings to chromosome territories: The role of topological constraints in genome folding, Rep. Prog. Phys. 77, 022601 (2014)

[25] M. Cates and J. Deutsch, Conjectures on the statistics of ring polymers, J. Phys. 47, 2121 (1986).

[26] S. K. Nechaev, A. N. Semenov, and M. K. Koleva, Dynamics of a polymer chain in an array of obstacles, Physica A 140, 506 (1987).

[27] D. Michieletto, On the tree-like structure of rings in dense solutions, Soft Matter 12, 9485 (2016).

[28] J. Smrek, K. Kremer, and A. Rosa, Threading of unconcatenated ring polymers at high concentrations: Double-folded vs time-equilibrated structures, ACS Macro Lett. 8, 155 (2019).

[29] D. Michieletto, D. Marenduzzo, E. Orlandini, G. Alexander, and M. Turner, Threading dynamics of ring polymers in a gel, ACS Macro Lett. 3, 255 (2014).
[30] E. Lee, S. Kim, and Y. Jung, Slowing down of ring polymer diffusion caused by inter-ring threading, Macromol. Rapid Commun. 36, 1115 (2015).

[31] D. Michieletto and M. S. Turner, A topologically driven glass in ring polymers, Proc. Natl. Acad. Sci. USA 113, 5195 (2016).

[32] D. Michieletto, N. Nahali, and A. Rosa, Glassiness and Heterogeneous Dynamics in Dense Solutions of Ring Polymers, Phys. Rev. Lett. 119, 197801 (2017).

[33] J. Smrek, I. Chubak, C. N. Likos, and K. Kremer, Active topological glass, Nat. Commun. 11, 26 (2020).

[34] Q. Huang, J. Ahn, D. Parisi, T. Chang, O. Hassager, S. Panyukov, M. Rubinstein, and D. Vlassopoulos, Unexpected Stretching of Entangled Ring Macromolecules, Phys. Rev. Lett. 122, 208001 (2019).

[35] D. Michieletto, D. Marenduzzo, E. Orlandini, G. P. Alexander, and M. S. Turner, Dynamics of self-threading ring polymers in a gel, Soft Matter 10, 5936 (2014).

[36] J. Smrek and A. Y. Grosberg, Minimal surfaces on unconcatenated polymer rings in melt, ACS Macro Lett. 5, 750 (2016).

[37] A. Hatcher and C. U. Press, Algebraic Topology (Cambridge University Press, Cambridge, UK, 2002).

[38] Y. Hiraoka, T. Nakamura, A. Hirata, E. G. Escolar, K. Matsue, and Y. Nishiura, Hierarchical structures of amorphous solids characterized by persistent homology, Proc. Nat. Acad. Sci. USA 113, 7035 (2016).

[39] T. Nakamura, Y. Hiraoka, A. Hirata, E. G. Escolar, and Y. Nishiura, Persistent homology and many-body atomic structure for medium-range order in the glass, Nanotechnology 26, 304001 (2015).

[40] K. Kremer and G. S. Grest, Dynamics of entangled linear polymer melts: A molecular-dynamics simulation, J. Chem. Phys. 92, 5057 (1990).

[41] A. E. Likhtman and M. Ponmurugan, Microscopic definition of polymer entanglements, Macromolecules 47, 1470 (2014).

[42] G. Kusano, K. Fukumizu, and Y. Hiraoka, Kernel method for persistence diagrams via kernel embedding and weight factor, J. Mach. Learn. Res. 18, 6947 (2017).

[43] M. E. Cates, Reptation of living polymers: Dynamics of entangled polymers in the presence of reversible chain-scission reactions, Macromolecules 20, 2289 (1987).

[44] A. Grosberg, Y. Rabin, S. Havlin, and A. Neer, Crumpled globule model of the three-dimensional structure of DNA, Europhys. Lett. 23, 373 (1993). 